\title{
Pseudopericardial effusion due to excess tension caused by a guide catheter during the MitraClip procedure
}

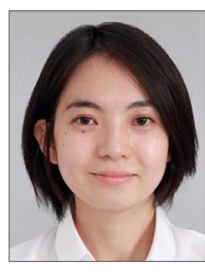

Ruri Ishibashi, MD; Akihisa Kataoka*, MD, PhD; Yusuke Watanabe, MD, PhD;

Ken Kozuma, MD, PhD

Department of Medicine, Division of Cardiology, Teikyo University School of Medicine, Tokyo, Japan

This paper also includes supplementary data published online at: www.asiaintervention.org

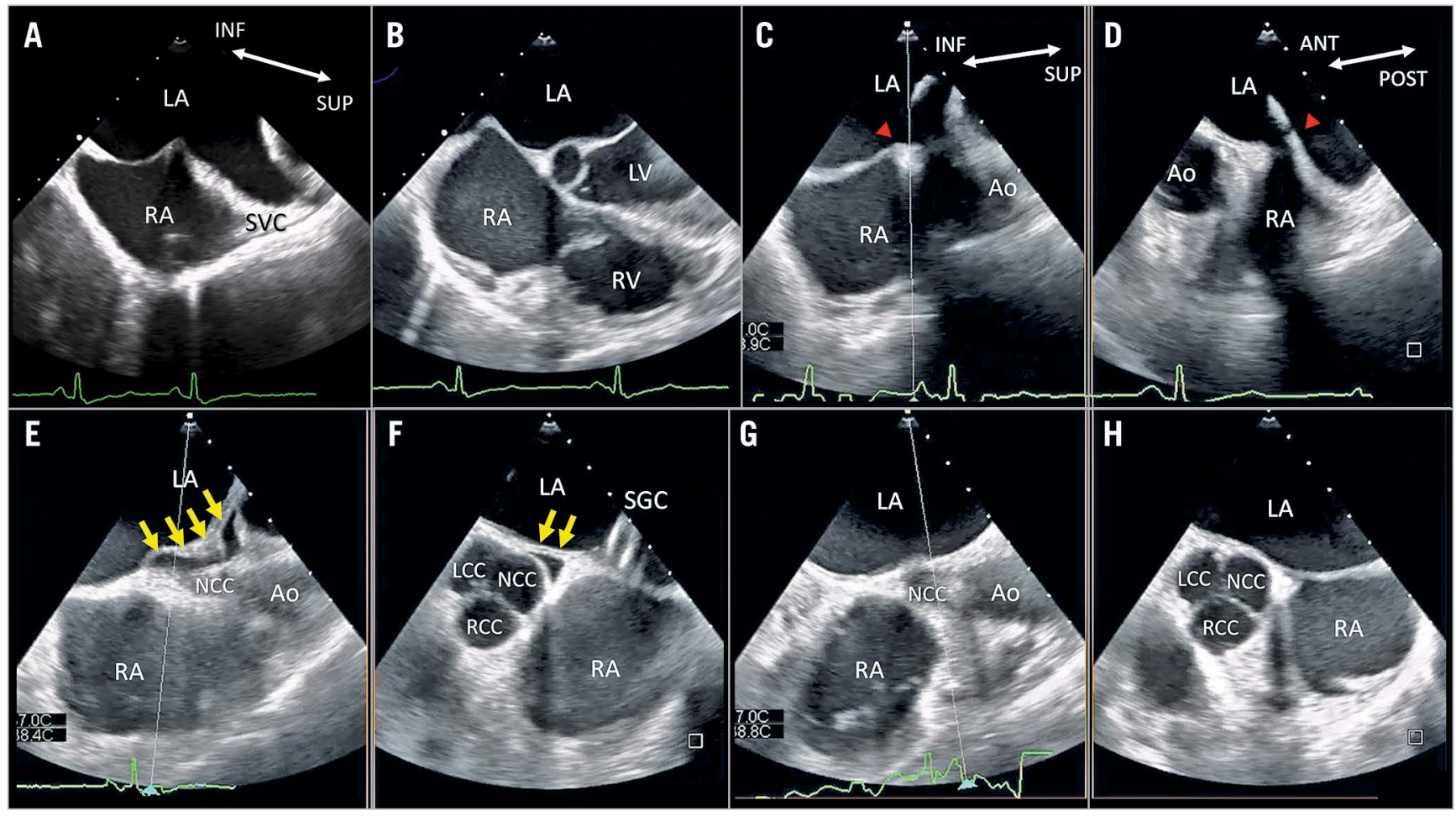

*Corresponding author: 2-11-1 Kaga, Itabashi-ku, Tokyo, 173-8606, Japan.

E-mail: kataoaki@sd5.so-net.ne.jp 
A 66-year-old man underwent transcatheter mitral valve repair for severe functional mitral regurgitation (MR) due to dilative cardiomyopathy.

There was no pericardial effusion (PE) (Panel A, Panel B, Moving image 1, Moving image 2), and transseptal puncture (TSP) was performed in the superior and anterior fossa ovalis without complications. Transoesophageal echocardiography (TEE) (EPIQ7/ X8-2t system; Philips, Andover, MA, USA) revealed that the steerable guide catheter (SGC) was pulling the osseous membrane and causing it to tent as it was inserted into the left atrial (LA) cavity (Panel C, Panel D, Moving image 3). A MitraClip G4 NTW $^{\circledR}$ (Abbott Vascular, Santa Clara, CA, USA) was successfully implanted once the MR had reduced. When the clip delivery system was removed from the SGC, TEE revealed a pericardial space between the LA wall and aortic root, indicating PE) (Panel E, Panel F, Moving image 4). No PE was observed elsewhere, and the vital signs remained unchanged, thereby excluding cardiac tamponade. The procedure was immediately terminated. However, the PE disappeared once the SGC was removed and did not return (Panel G, Panel H, Moving image 5, Moving image 6).

PE between the LA wall and aortic root is common. This fatal complication of TSP stems from improper puncture of the fossa ovalis $^{1}$. However, we detected no such punctures using TEE. There is a pericardial sac, which has a double-walled structure, surrounded by the LA, right atrium, and base of the aortic root. We suspected that the redundant LA wall and pericardium were pulled due to excess tension generated by the SGC and displaced in the direction of the LA cavity, thereby creating free space. The Heart
Team should consider pseudo-PE when excess tension is generated by the SGC during the MitraClip procedure.

\section{Conflict of interest statement}

The authors have no conflicts of interest to declare.

\section{Reference}

1. Salghetti F, Sieira J, Chierchia GB, Curnis A, de Asmundis C. Recognizing and reacting to complications of trans-septal puncture. Expert Rev Cardiovasc Ther. 2017;15:905-12.

\section{Supplementary data}

Moving image 1 \& Moving image 2. Bi-caval, four-chamber, and live xPlane images (bi-caval and short-axis views) of TEE performed during the MitraClip procedure. Transseptal puncture was performed in the superior and anterior fossa ovalis without complications.

Moving image 3. At the insertion of the SGC, the tenting of the intraseptal wall is shown.

Moving image 4. After the removal of the clip delivery system, the pericardial effusion between the LA wall and aortic root is shown.

Moving image 5 \& Moving image 6. After the removal of the $\mathrm{SGC}$, the pericardial effusion immediately disappeared.

The supplementary data are published online at: www.asiaintervention.org 